Pacific

Journal of

Mathematics

IRREDUCIBILITY OF -1-CLASSES ON ANTICANONICAL RATIONAL SURFACES AND FINITE GENERATION OF THE EFFECTIVE MONOID

Mustapha LAHYANE AND BRIAN HARBOURNE 


\title{
IRREDUCIBILITY OF -1-CLASSES ON ANTICANONICAL RATIONAL SURFACES AND FINITE GENERATION OF THE EFFECTIVE MONOID
}

\author{
Mustapha LAHYANE AND BRIAN HARBOURNE
}

\begin{abstract}
We give necessary and sufficient conditions for a divisor class on smooth projective anticanonical rational surfaces to be the class of a smooth rational curve of self-intersection $\mathbf{- 1}$. We characterize smooth projective anticanonical rational surfaces for which the monoid of classes (modulo algebraic equivalence) of effective divisors is not finitely generated, extending results of Lahyane for the case of rational surfaces $X$ with $K_{X}^{2}=0$.
\end{abstract}

\section{Introduction}

A surface will always mean a smooth projective variety of dimension 2, over an arbitrary algebraically closed field. We do not require the characteristic to be 0 .

For a nonrational surface $X$, the number of irreducible exceptional curves (irreducible curves $C$ satisfying $C^{2}=K_{X} \cdot C=-1$, which we will also call -1-curves) is always finite. If $X$ is given as a blow-up of points on a nonrational minimal model $Y$, it is easy to identify all -1 -curves $C$ on $X$. When $X$ is rational, this is no longer true: there can be infinitely many -1 -curves on $X$, and there can be infinitely many divisor classes $C$ that satisfy $C^{2}=K_{X} \cdot C=-1$ and are not the class of an irreducible curve, or even of an effective divisor. Thus it is desirable to have a criterion on rational surfaces for when a divisor class $C$ satisfying $C^{2}=K_{X} \cdot C=-1$ is the class of a -1 -curve. We give such a necessary and sufficient criterion for certain rational surfaces $X$ : first in case $K_{X}^{2} \geq 0$ (Proposition 3.3) and then (Theorem 3.5) more generally for any anticanonical rational surface, by which we mean a surface $X$ for which the anticanonical class $-K_{X}$ is the class of an effective divisor. See [Harbourne 1997] for more information about anticanonical rational surfaces, and Theorem 4.1 of [Alberich-Carramiñana] for an alternate independent characterization of -1-curves.

We also consider the related question of when the monoid $M(X)$ (in the group of divisors modulo algebraic equivalence) of classes of effective divisors on an anticanonical rational surface is finitely generated. The behavior of $M(X)$ can

Lahyane was supported by a Marie Curie grant number HPMD-GH-01-00097-01 at the department "Álgebra, Geometría y Topología" of Valladolid University, Spain. Harbourne was supported by a grant from NSA. 
vary widely among surfaces generally; see for instance [Rosoff 1981] and [Rosoff 2002, Theorems 1-4]. In the case of a rational surface $X$, for example, whereas $M(X)$ can fail to be finitely generated, $M(X)$ is finitely generated if $K_{X}^{2}>0$ (see Proposition 4.3(a)), or, by [Lahyane 2001a], if $K_{X}^{2}=0$ and the anticanonical divisor $-K_{X}$ of $X$ is not nef (numerically effective - see Definition 2.6).

Given a surface $X$, a sufficient condition for $M(X)$ not to be finitely generated is that $X$ have an infinite number of integral curves of negative self-intersection [Rosoff 1980, Fact, p. 426]. If $X$ is an anticanonical rational surface, we show that this condition is also necessary. In fact, if $X$ is rational and anticanonical, we show in Corollary 4.2 that $M(X)$ is not finitely generated if and only if $X$ contains infinitely many irreducible curves $C$ of self-intersection $-2 \leq C^{2} \leq-1$.

Although a rational surface with $K_{X}^{2} \geq 0$ is always anticanonical, our results on finite generation of $M(X)$ are of interest mainly in the case of anticanonical rational surfaces $X$ with $K_{X}^{2}<0$, since $M(X)$ is always finitely generated when $K_{X}^{2}>0$, and [Lahyane 2001a; 2001b; 2004] give a complete characterization when $K_{X}^{2}=0$ (see Proposition 4.3(c, d)). This characterization is in terms of the set of -2-curves, which in this situation must be a finite set. We partially extend this result by showing (see Proposition 4.3(e)) for rational surfaces $X$ such that $-K_{X}$ is the class of a reduced irreducible curve of negative self-intersection, that $M(X)$ is finitely generated if and only if the set of classes of -2-curves is both finite and spans $K_{X}^{\perp}$.

We close the paper with several examples of anticanonical rational surfaces for which $M(X)$ is not finitely generated, applying our results to give explicit infinite families of -1 -curves.

\section{Preliminaries}

In this section, we recall the notions that we need and set our notation and terminology. We refer to [Hartshorne 1977] as a general reference. Let $X$ be a surface. A divisor on $X$ is effective if it is a nonnegative linear combination of prime divisors (i.e., reduced irreducible curves). Similarly, we say a divisor class (modulo algebraic equivalence) on $X$ is effective if this class contains an effective divisor. If $X$ is rational, note that algebraic, linear, numerical and rational equivalence of divisors on $X$ are the same, and that $\operatorname{Pic}(X)$ is isomorphic to the group $\mathrm{Cl}(X)$ of divisors modulo linear equivalence. The latter is a finitely generated free abelian group; its rank is referred to as the Picard number $\rho(X)$ of $X$.

We being by recalling Riemann-Roch's theorem in the case of rational surfaces.

Theorem 2.1. Let $D$ be a divisor on a rational surface $X$. Then

$$
h^{0}\left(X, \mathscr{O}_{X}(D)\right)-h^{1}\left(X, \mathscr{O}_{X}(D)\right)+h^{2}\left(X, \mathscr{O}_{X}(D)\right)=1+\frac{1}{2}\left(D^{2}-K_{X} \cdot D\right)
$$

and

$$
h^{2}\left(X, \mathscr{O}_{X}(D)\right)=h^{0}\left(X, \mathscr{O}_{X}\left(K_{X}-D\right)\right) .
$$


In the next lemma, $K_{Y}^{\perp}$ denotes the subgroup of $\mathrm{Cl}(Y)$ of classes $x$ on a surface $Y$ with $x \cdot K_{Y}=0$.

Lemma 2.2. Let $Y$ be a rational surface such that $K_{Y}^{2} \geq 0$, and let $x \in K_{Y}^{\perp}$. Then $Y$ is anticanonical and, if $x^{2} \geq 0$, then $x^{2}=0$ and $x=p K_{Y}$ for some integer $p$. In particular, if $K_{Y}^{2}>0$, then $x$ and $p$ vanish.

Proof. Since $Y$ is rational, we have $h^{0}\left(Y, O_{Y}\left(2 K_{Y}\right)\right)=0$; now by Theorem 2.1 it follows that $Y$ is anticanonical. If $x^{2}>0$, then $x^{\perp}$ is negative definite by the Hodge index theorem, which contradicts $K_{Y}^{2} \geq 0$. Thus $x^{2}=0$. Now for any $y \in K_{Y}^{\perp}$, we have $(t x+y)^{2}>0$ for some integer $t$ unless $x \cdot y=0$. Thus either $x=0$ or $x^{\perp}=K_{Y}^{\perp}$, and hence, since $K_{Y}$ is primitive (unless $Y$ is minimal, in which case $x \in K_{Y}^{\perp}$ with $x^{2}=0$ implies $x=0$ ), $x=p K_{Y}$ for some integer $p$.

Definition 2.3. We call a divisor $D$ on a rational surface $Y$ a -1-divisor if $D^{2}=$ $D \cdot K_{Y}=-1$, and we call a divisor class on $Y$ a -1-class if it is the class of a -1-divisor. We call a-1-divisor a -1-curve if it is effective and irreducible (and necessarily therefore reduced). Similarly, we call a divisor $D$ on a rational surface $Y$ a -2-divisor if $D^{2}=-2$ and $D \cdot K_{Y}=0$, and we call a divisor class on $Y$ a -2-class if it is the class of a-2-divisor. We call a-2-divisor a-2-curve if it is effective and irreducible (and necessarily therefore reduced).

Remark 2.4. Let $C$ be a prime divisor on a surface $X$. By the adjunction formula, $C^{2}+C \cdot K_{X}=2 g-2$, where $g$ is the arithmetic genus of $C$. It follows that a -1 - or -2-curve is smooth and rational, and, if $X$ is an anticanonical rational surface, that $C^{2}<0$ implies either $-K_{X} \cdot C<0$ and hence $C$ is a fixed component of $\left|-K_{X}\right|$ (of which there are at most finitely many), or $-K_{X} \cdot C \geq 0$ and $C$ is a -1 or -2 -curve.

Remark 2.5. Not every rational surface has any -1-divisors or -2-divisors. For instance $\mathbb{P}^{2}$ has neither and the Hirzebruch surface $\mathbb{F}_{n}$ does not have -1-divisors when $n$ is even, or -2-divisors when $n$ is odd. However, if a rational surface $Y$ has $\rho(Y) \geq 3$, the set of -1 -curves on $Y$ is not empty, while if $\rho(Y) \geq 10$, there are always infinitely many -1 -divisors and -2 -divisors. Moreover, whereas every -1-curve is a-1-divisor, and likewise for -2-curves, the converse is not true in general. For example, consider the Hirzebruch surface $\mathbb{F}_{2 n+1}$ with $n>0$. Let $B$ denote the base curve (i.e., the integral curve of self-intersection $-(2 n+1))$ and let $F$ be any fiber of the ruling on $\mathbb{F}_{2 n+1}$. Then $B+n F$ is a -1 -class but contains no -1 -curve, since $B$ is a fixed component of $|B+n F|$. Similarly, for $F_{2 n}$ with $n>1, B+(n-1) F$ is a -2 -class but contains no -2-curve. For another example, let $X$ be obtained by blowing up $\mathbb{P}^{2}$ at 10 points. Then $K_{X}$ is a -1-class, but it is not effective, and hence not the class of any -1-curve. Finally, let $E_{1}$ and $E_{2}$ be the exceptional curves obtained by blowing up two distinct points of a rational surface. Then $E_{1}-E_{2}$ is a -2-class and is not the class of any -2-curve.

Definition 2.6. A divisor or divisor class $D$ on a surface $X$ is said to be numerically effective (nef for short) if $D \cdot C \geq 0$ for every prime divisor $C$ on $X$. 
Example 2.7. An effective divisor that meets each of its irreducible components nonnegatively is nef. Hence prime divisors of nonnegative self-intersection are nef, as is the class of a line pulled back to $X$ via $\pi$, where $\pi: X \rightarrow \mathbb{P}^{2}$ is the blow-up of the projective plane $\mathbb{P}^{2}$ at a finite set of points.

Remark 2.8. We recall some notions in the case that we have a birational morphism $X \rightarrow \mathbb{P}^{2}$ [Harbourne 1985b]. Then $X$ is obtained by blowing up a sequence of points of $\mathbb{P}^{2}$, possibly infinitely near. More precisely, there are points $p_{i} \in X_{i-1}$ such that $p_{1} \in X_{0}=\mathbb{P}^{2}$ and each $X_{i} \rightarrow X_{i-1}$, for $i \geq 1$, is obtained recursively by blowing up $p_{i} \in X_{i-1}$. Then $X=X_{r}$, where $r=\rho(X)-1$. Also, we set $E_{i}$ to be the class of the scheme theoretic inverse image of $p_{i}$ under the composition $X \rightarrow X_{i-1}$, and we take $E_{0}$ to be the pullback to $X$ of the class of a line in $X_{0}$. Note that $E_{i}$ is an effective -1-class for $i>0$, that $E_{r}$ is always the class of a-1-curve, and that $-K_{X}=3 E_{0}-E_{1}-\cdots-E_{r}$. The classes $E_{i}$, for $i \geq 0$, give an orthogonal basis of $\mathrm{Cl}(X)$, called an exceptional configuration, such that $-E_{0}^{2}=E_{1}^{2}=\cdots=-1$. Every birational morphism $X \rightarrow \mathbb{P}^{2}$ is associated to an exceptional configuration, which is unique up to the order of the $E_{i}, i>0$. We also recall the action of the Weyl group on $\mathrm{Cl}(X)$. Define classes $s_{0}=E_{0}-E_{1}-E_{2}-E_{3}, s_{1}=E_{1}-E_{2}$, $s_{2}=E_{2}-E_{3}, \ldots, s_{r-1}=E_{r-1}-E_{r}$. Reflections $\sigma_{i}: x \mapsto x+\left(x \cdot r_{i}\right) r_{i}$ in these classes generate a group of isometries of $\mathrm{Cl}(X)$ under whose action $K_{X}$ is fixed; this is called the Weyl group and denoted $W_{r}$. A useful fact for any class $D$ with $D \cdot s_{i} \geq 0$ for all $i$ is that $w D=D+N$ for any $w \in W_{r}$, where $N$ is a nonnegative integer linear combination of the classes $s_{i}$. See [Harbourne 1985a, Lemma 0.9] or [Looijenga 1981, I.3.3].

\section{A criterion for -1-curves}

We first consider the case that $X$ is a rational surface with $K_{X}^{2} \geq 0$. If $X$ has $\mathbb{P}^{2}$ as a minimal model, the following lemma shows that every -1 -class on $X$ is effective.

Lemma 3.1. Assume that there is a birational morphism $X \rightarrow \mathbb{P}^{2}$ and that $K_{X}^{2} \geq 0$. If $E$ is $a-1$-class on $X$, then $E$ is effective.

Proof. Consider a given exceptional configuration $E_{i}, 0 \leq i \leq \rho(X)-1$, corresponding to $X \rightarrow \mathbb{P}^{2}$. By blowing up additional points if need be, we may assume that $K_{X}^{2}=0$ (i.e., $\rho(X)=10$ ), since effectivity is preserved under pullback, as is the expression of a pullback in terms of the $E_{i}$. If $E$ is any -1-class, then $E-E_{9} \in K_{X}^{\perp}$; hence we can write $E=E_{9}-r K_{X}+N$ for some $N \in K_{X}^{\perp} \cap E_{9}^{\perp}$, in which case $r=-N^{2} / 2$. Thus the -1 -classes are precisely the classes of the form $E_{9}-r K_{X}+N$ with $N \in K_{X}^{\perp} \cap E_{9}^{\perp}$ and $r=-N^{2} / 2$. One can check that the classes of the given form $E_{9}-r K_{X}+N$ form the orbit $W_{9} E_{9}$ of $E_{9}$ under the action of $W_{9}$ (using the fact from [Kac 1985] that $W_{9}$ contains all homomorphisms of the form $\tau_{s}: x \mapsto x+\left(x \cdot K_{X}\right) s+m K_{X}$, where $s \in K_{X}^{\perp}$ and $m$ is the uniquely determined integer such that $\left.x^{2}=\left(x+\left(x \cdot K_{X}\right) s+m K_{X}\right)^{2}\right)$. Thus, by Remark 2.8, $E=E_{9}+N^{\prime}$ 
for some nonnegative linear combination $N^{\prime}$ of the classes $s_{i}$; hence $E \cdot E_{0} \geq 0$. But by Riemann-Roch, either $|E|$ or $\left|K_{X}-E\right|$ must be nonempty, and since we now see that $\left(K_{X}-E\right) \cdot E_{0} \leq-3$, the fact that $E_{0}$ is nef implies $E$ is effective.

The next result extends Lemma 3.1 to rational surfaces $X$ with $K_{X}^{2} \geq 0$ but which need not have a birational morphism to $\mathbb{P}^{2}$.

Lemma 3.2. Let $X$ be a rational surface such that $K_{X}^{2} \geq 0$. If $E$ is a-1-class on $X$, then $E$ is effective.

Proof. This is just Lemma 3.1 when $X$ is a blow-up of points of $\mathbb{P}^{2}$, so say $X$ is a blow up of points on a Hirzebruch surface $\mathbb{F}_{n}$. Let $B$ be the base curve on $\mathbb{F}_{n}$ (so $B^{2}=-n$ ), and let $F$ be a fiber in the ruling on $\mathbb{F}_{n}$. Blow up more points, if need be, so that $K_{X}^{2}=0$ (so now $X$ is a blow-up of $\mathbb{F}_{n}$ at 8 points, which are allowed to be infinitely near; let $E_{1}, \ldots, E_{8}$ be the corresponding exceptional divisors). Since a blow-up of $\mathbb{F}_{n}$ at any point $p$ can, by an elementary transformation, be regarded as a blow-up of $\mathbb{F}_{n+1}$ (if $p \in B$ ) or of $\mathbb{F}_{n-1}$ (if $p$ is not on $B$ ), we can assume $n$ is even. Since $X$ would be a blow-up of $\mathbb{P}^{2}$ if $n<2$ we may also assume that $n \geq 2$. Since $n$ is even, there is an isometric isomorphism $f: \operatorname{Cl}(X) \rightarrow G$, where $G$ is the free abelian group on elements $e_{0}, \ldots, e_{9}$, endowed with the intersection product one would expect if it were an exceptional configuration. This isomorphism is given by $f\left(E_{i}\right)=e_{i+1}$ for $2 \leq i \leq 8, f\left(E_{1}\right)=e_{0}-e_{1}-e_{2}, f(F)=e_{0}-e_{1}$, and $f(B)=e_{0}-n\left(e_{0}-e_{1}\right) / 2-e_{2}$. Note that $f$ maps $s_{0}^{\prime}=E_{1}-E_{2}, s_{1}^{\prime}=B+(n-2) F / 2$, $s_{2}^{\prime}=F-E_{1}-E_{2}, s_{3}^{\prime}=E_{2}-E_{3}, \ldots, s_{8}^{\prime}=E_{7}-E_{8}$, respectively, to the elements $s_{0}, \ldots, s_{8}$ (defined as in Remark 2.8, but using $e_{i}$ instead of $E_{i}$ ). Any -1 -class on $X$ is of the form $E_{8}+N^{\prime}$, where $N^{\prime}$ is a nonnegative linear combination of the $s_{i}^{\prime}$ (since $f\left(E_{8}+N^{\prime}\right)=e_{9}+N$ is a -1 -class in terms of the $e_{i}$, and as in the proof of Lemma $3.1, N$ is a nonnegative linear combination of the $\left.s_{i}\right)$. Since $(B+n F) \cdot s_{i}^{\prime} \geq 0$ for all $i$, it follows that $(B+n F) \cdot E=(B+n F) \cdot\left(E_{8}+N^{\prime}\right) \geq 0$. But $B+n F$ is nef and $(B+$ $n F) \cdot K_{X}=-2-n$. As in the proof of Lemma 3.1, either $E$ or $K_{X}-E$ is effective, and since $(B+n F) \cdot\left(K_{X}-E\right) \leq-n-2<0$, it can only be $E$ that is effective.

Now we give a characterization for a -1-class to be the class of a -1-curve.

Proposition 3.3. Let $X$ be a rational surface such that $K_{X}^{2} \geq 0$. Let $E$ be a -1-class on $X$. Then $E$ is a-1-curve if and only if two conditions hold:

(1) $E \cdot C \geq 0$ for all fixed components $C \neq E$ of the complete linear system $\left|-K_{X}\right|$.

(2) $E \cdot C \geq 0$ for all-2-curves $C$ on $X$.

Proof. The forward implication is clear, so say $E$ is a -1-class. By Lemma 3.2, $E$ is effective, but $E^{2}<0$, so there must exist a prime divisor $C$ with $C^{2}<0$ and $E \cdot C<0$. By Remark 2.4, and assuming (1) and (2) are satisfied, $C$ must be a -1-curve; hence we get $(E-C) \cdot K_{X}=0$ and $(E-C)^{2} \geq 0$. It follows by Lemma 2.2 that $(E-C)^{2}=0$ (hence $E \cdot C=-1$ ) and $E-C=p K_{X}$ for some integer $p$, 
with $p=0$ if $K_{X}^{2}>0$ (in which case $E=C$ ). If however $K_{X}^{2}=0$, the fact that $E \cdot\left(C+p K_{X}\right)=E^{2}=-1$ again implies that $p=0$ and hence $E=C$.

To extend Proposition 3.3 to the case of anticanonical rational surfaces $X$ with $K_{X}^{2}<0$, we need a lemma.

Lemma 3.4. Let $X$ be a rational surface. Let $E$ be an effective -1-class for which $K_{X}-E$ and all $l K_{X}+E$, for $l>0$, are not effective. Then $E \cdot C \geq 0$ for all -1 -curves $C \neq E$.

Proof. First assume that there is a birational morphism $X \rightarrow \mathbb{P}^{2}$, and hence an exceptional configuration $E_{0}, \ldots, E_{n}$. Note that $X$ is a specialization of a surface $X^{\prime}$ obtained by blowing up generic points of $\mathbb{P}^{2}$. If $\rho(X)<10$, the set of all -1-classes is listed in [Manin 1986], and given any -1-classes $E$ and $C$, one directly checks that $E \cdot C \geq 0$ as long as $E \neq C$. Thus we may assume that $n \geq 10$. With respect to the blow-up $X^{\prime} \rightarrow \mathbb{P}^{2}$ we have the exceptional configuration $E_{0}^{\prime}, \ldots, E_{n}^{\prime}$, which specializes to $E_{0}, \ldots, E_{n}$. We can define an isometric isomorphism $f: \mathrm{Cl}\left(X^{\prime}\right) \rightarrow \mathrm{Cl}(X)$ by $f\left(E_{i}^{\prime}\right)=E_{i}$ for all $i$, with the inverse denoted by '. By semicontinuity we have $f\left(M\left(X^{\prime}\right)\right) \subset M(X)$, so $l K_{X}^{\prime}+E^{\prime}=l K_{X^{\prime}}+E^{\prime}$ and $K_{X}^{\prime}-E^{\prime}=K_{X^{\prime}}-E^{\prime}$ are not effective. It follows by Riemann-Roch that $E^{\prime}$ is effective. Since for any -1-curve $C$ on $X, C^{\prime}$ is a-1-curve on $X^{\prime}$ (since $f\left(D^{\prime}\right)$ is a component of $C$ for any component $D^{\prime}$ of $C^{\prime}$ ), it is enough to prove the lemma on $X^{\prime}$. Thus we reduce to the case that $X$ is obtained by blowing up generic points, and so hereafter we suppress the primes.

Since $X$ is generic, the action of $W_{n}$ preserves $M(X)$. (To see this, consider an effective class $F$. Since the exceptional configuration $E_{0}, \ldots, E_{n}$ comes from blowing up generic points, $w\left(E_{0}\right), \ldots, w\left(E_{n}\right)$ is another exceptional configuration for $X$ for each $w \in W_{n}$ [Nagata 1960b], and since $w$ is invertible $w\left(E_{0}\right), \ldots, w\left(E_{n}\right)$ also comes from blowing up generic points. If $F=\sum_{i \geq 0} a_{i} E_{i}$, then $w(F)=$ $\sum_{i \geq 0} a_{i} w\left(E_{i}\right)$, so both $F$ and $w(F)$ have the same coefficients with respect to exceptional configurations coming from blowing up generic points. Hence $F$ is effective if and only if $w(F)$ is.)

Since $E$ (and hence $w(E)$ for each $w$ ) is effective, we always have $w(E) \cdot E_{0} \geq 0$. Thus we can choose $w^{\prime} \in W_{n}$ such that $w^{\prime}(E) \cdot E_{0}$ is a minimum, and hence $w^{\prime}(E)$ meets $s_{0}$ nonnegatively (because otherwise $\left.\sigma_{0} w^{\prime}(E) \cdot E_{0}<w^{\prime}(E) \cdot E_{0}\right)$. Moreover, $W$ includes the group of permutations of $E_{1}, \ldots, E_{n}$ (just consider the action by the $\sigma_{i}$ for $i>0$ ), so we can apply a permutation $\sigma$ such that $\sigma w(E) \cdot s_{i} \geq 0$ for $i>0$, and we still have $\sigma w^{\prime}(E) \cdot s_{0} \geq 0$, since otherwise $\sigma_{0} \sigma w^{\prime}(E) \cdot E_{0}<w^{\prime}(E) \cdot E_{0}$. Thus for some $w \in W_{n}$ we have $w(E) \cdot s_{i} \geq 0$ for all $i \geq 0$ (the algorithm of [Harbourne $1985 \mathrm{~b}$ ] gives an effective method for finding such an element $w$ ), and this implies that $w(E)$ must be a nonnegative integer linear combination of the classes $-K_{X}$, $E_{1}+\cdots+E_{n}, E_{2}+\cdots+E_{n}, \ldots, E_{n}, E_{0}, E_{0}-E_{1}$, and $2 E_{0}-E_{1}-E_{2}$ (compare 
[Harbourne 1985b, Lemma 1.4]), each of which other than $-K_{X}$ is effective, and each of which other than $-K_{X}$ and $E_{n}$ meets $-K_{X}$ at least twice.

Since $l K_{X}+E$ (and hence $\left.w\left(l K_{X}+E\right)=l K_{X}+w(E)\right)$ is not effective, this linear combination does not involve $-K_{X}$. Since $-K_{X} \cdot w(E)=w\left(-K_{X}\right) \cdot w(E)=$ $-K_{X} \cdot E=1$, the only possibility is $w(E)=E_{n}$. Since $X$ is generic and $n>2$, the set of -1-curves (see [Nagata 1960b]) is precisely the orbit $W_{n} E_{n}$, so $E$ and $C$ are both -1 -curves, so if $E \neq C$, then $E \cdot C \geq 0$.

We are left with handling the case that there is no birational morphism to the plane. By blowing up enough additional generic points on $X$ (and thus off any given $C$ ), we may assume that $C$ is still a -1 -curve and that $X$ is obtained by blowing up at least $n-1$ generic points of an $\mathbb{F}_{n}$, for some $n$. By elementary transformations at these blow-ups, there is a birational morphism $X \rightarrow \mathbb{P}^{2}$. After pulling $E$ back to the blow-up, it is still an effective -1 -class, and $l K_{X}+E$ and $K_{X}-E$ are still not effective, so we are reduced to the previous case.

Now we state our characterization for a -1-divisor on an anticanonical rational surface to be irreducible.

Theorem 3.5. Let $X$ be an anticanonical rational surface and $E$ a-1-class on $X$. Then $E$ is $a-1$-curve if and only if the following three assertions hold:

(1) $E \cdot C \geq 0$ for all fixed components $C \neq E$ of $\left|-K_{X}\right|$.

(2) $E \cdot C \geq 0$ for all-2-curves $C$ on $X$.

(3) Neither $K_{X}+E$ nor $K_{X}-E$ is effective.

Proof. The forward implication is clear, except perhaps for why $K_{X}+E$ is not effective when $E$ is a -1-curve. But let $X \rightarrow Y$ be obtained by blowing down $E$. Let $A$ be the pullback to $X$ of an ample divisor on $Y$. Note that $A \cdot E=0$, that $A$ is nef, and that

$$
A \cdot\left(K_{X}+E\right)=A \cdot K_{X}=A \cdot\left(K_{Y}+E\right)=A \cdot K_{Y}
$$

is negative since $A$ is the pullback of an ample divisor on $Y$ and $-K_{Y}$ is effective (since on $X$ we have $-K_{Y}=-K_{X}+E$, which is effective), so $K_{X}+E$ is not effective.

For the converse, $K_{X}-E$ is not effective by assumption, so it follows by Riemann-Roch that $E$ is effective. As in the proof of Proposition 3.3, $E \cdot C<0$ for some -1-curve $C$ on $X$. But $l K_{X}+E$ is not effective for $l>0$. (Suppose it were effective. Then, since $-K_{X}$ is effective, $\left(l K_{X}+E\right)-(l-1) K_{X}=K_{X}+E$ would be too, contrary to hypothesis.) Thus we can apply Lemma 3.4, and hence $E \cdot C \geq 0$ unless $E=C$.

Remark 3.6. The fact that, for example, $\left(3 E_{0}-E_{1}-E_{2}-\cdots-E_{10}\right)+2 E_{10}$, $-\left(3 E_{0}-E_{1}-\cdots-E_{10}\right)$ and $6 E_{0}-2\left(E_{1}+\cdots+E_{8}\right)-\left(E_{9}+E_{10}+E_{11}\right)+$ $E_{12}+E_{13}$ are -1 -classes that cannot be classes of -1-curves shows the necessity of hypothesis (3) in Theorem 3.5. 


\section{Criteria for finite generation of $M(X)$}

In this section we present some results on finite generation of the monoid $M(X)$ of effective divisor classes on an anticanonical rational surface $X$. For $\rho(X)<3$, finite generation of $M(X)$ is well known. For example, if $\rho(X)=1$, then $X$ is $\mathbb{P}^{2}$ (in which case $M(X)$ is generated by the class of a line). If $\rho(X)=2$, then $X$ is a Hirzebruch surface $\mathbb{F}_{n}$ (in which case $M(X)$ is generated by the classes $B$ of the base curve and $F$ of a fiber of the ruling). The following lemma gives generators for the case $\rho(X)>2$.

Lemma 4.1. Let $\mathcal{N}(X)$ be the set of prime divisors of negative self-intersection on an anticanonical rational surface $X$ with $\rho(X) \geq 3$. The monoid $M(X)$ of effective classes is generated by $\mathcal{N}(X)$ together with $-K_{X}$.

Proof. It is enough to show that the class $D$ of every prime divisor is in the submonoid of $\mathrm{Cl}(X)$ generated by $\mathcal{N}(X)$ and $-K_{X}$. If $D^{2}<0$, then $D \in \mathcal{N}(X)$. So assume $D^{2} \geq 0$; hence $D$ is nef. Then it is enough by induction to show that $D-C$ is effective for some $C \in \mathcal{N}(X)$ or for $C=-K_{X}$.

First assume that there is a birational morphism $X \rightarrow \mathbb{P}^{2}$, so we have an exceptional configuration $E_{0}, \ldots, E_{n}$ and we can write $D=m_{0} E_{0}-m_{1} E_{1}-\cdots-m_{n} E_{n}$ for some (nonnegative) integers $m_{i}$.

As in the proof of Lemma 3.4, we consider the blow-up $X^{\prime} \rightarrow \mathbb{P}^{2}$ at generic points, for which we write the exceptional configuration as $E_{0}^{\prime}, \ldots, E_{n}^{\prime}$, and we have the isometry $f: \mathrm{Cl}\left(X^{\prime}\right) \rightarrow \mathrm{Cl}(X)$ defined by $f\left(E_{i}^{\prime}\right)=E_{i}$ for all $i$, with the inverse to $f$ being denoted by'; by semicontinuity we again have $f\left(M\left(X^{\prime}\right)\right) \subset M(X)$.

Now, $D^{\prime 2}=D^{2} \geq 0,-K_{X^{\prime}} \cdot D^{\prime}=-K_{X} \cdot D \geq 0$, and $E_{0} \cdot D \geq 0$ (hence $K_{X}-D$ and so $K_{X^{\prime}}-D^{\prime}$ are not effective), so $D^{\prime}$ is effective by Riemann-Roch. As in the proof of Lemma 3.4, for some $w \in W_{n}, w\left(D^{\prime}\right)$ must be a nonnegative integer linear combination of the classes $-K_{X}^{\prime}, E_{1}^{\prime}+\cdots+E_{n}^{\prime}, E_{2}^{\prime}+\cdots+E_{n}^{\prime}, \ldots, E_{n}^{\prime}$, $E_{0}^{\prime}=\left(E_{0}^{\prime}-E_{1}^{\prime}-E_{2}^{\prime}\right)+E_{1}^{\prime}+E_{2}^{\prime}, E_{0}^{\prime}-E_{1}^{\prime}=\left(E_{0}^{\prime}-E_{1}^{\prime}-E_{2}^{\prime}\right)+E_{2}^{\prime}$, and $2 E_{0}^{\prime}-E_{1}^{\prime}-E_{2}^{\prime}=$ $2\left(E_{0}^{\prime}-E_{1}^{\prime}-E_{2}^{\prime}\right)+E_{1}^{\prime}+E_{2}^{\prime}$. The class $f\left(w^{-1}\left(-K_{X}^{\prime}\right)\right)=-K_{X}$ is effective by hypothesis. If $F^{\prime}$ is any other of the listed classes, then $F^{\prime}$ is effective, hence (as in the proof of Lemma 3.4) so is $w^{-1}\left(F^{\prime}\right)$, and therefore also $f\left(w^{-1}\left(F^{\prime}\right)\right)$. Thus, if $-K_{X}^{\prime}$ occurs in the combination, $D+K_{X}$ is effective. If one of the other listed classes occurs, $D-E$ is effective for some effective -1 -class $E$ (since each of the other listed classes is a sum of -1-curves, and the action of $W_{n}$ on $X^{\prime}$ preserves the set of -1-curves). We do not know that all of the components of $E$ have negative self-intersection, but since $E^{2}<0$, we know at least one of them must. Take $C$ to be a component of $E$ of negative self-intersection; then $D-C=(D-E)+(E-C)$ is effective. Thus in any case we conclude by induction that $D$ is in the monoid generated by $\mathcal{N}(X)$ together with $-K_{X}$.

Now assume that there is no birational morphism $X \rightarrow \mathbb{P}^{2}$. Thus a minimal model for $X$ must be $\mathbb{F}_{n}$ for some $n$. Since $\rho(X) \geq 3, X$ would be a blow-up of 
$\mathbb{P}^{2}$ unless $n \geq 2$, so we may assume $n \geq 2$. In any case, $X$ is obtained by blowing up $r=\rho(X)-2>0$ points $p_{i}$ of $\mathbb{F}_{n}$, the corresponding exceptional divisors being $E_{1}, \ldots, E_{r}$.

If $r=\rho(X)-2 \leq n-2$, write $D=b B+c F-m_{1} E_{1}-\cdots-m_{r} E_{r}$ for some integers $b, c$, and $m_{i}$. Since $D$ is nef, we know $D \cdot E_{i}=m_{i} \geq 0, D \cdot F=b \geq 0$, $D \cdot\left(F-E_{i}\right)=b-m_{i} \geq 0$, and $D \cdot B=c-b n \geq 0$. Thus $c \geq b n \geq m_{1}+\cdots+m_{r}$, so $k=c-\left(m_{1}+\cdots+m_{r}\right) \geq 0$; hence we can write $D=b B+k\left(F-E_{1}\right)+$ $k E_{1}+m_{1}\left(F-E_{1}\right)+\cdots+m_{r}\left(F-E_{r}\right)$ as a sum of effective divisors of negative self-intersection. Letting $C$ be a component of negative self-intersection of one of the summands that has a positive coefficient, we see $D-C$ is effective, as desired.

If, however, $r=\rho(X)-2>n-2$, let $X^{\prime \prime}$ be the surface obtained by blowing up $r$ generic points $p_{i}^{\prime \prime}$ of $\mathbb{F}_{n}$, with the corresponding exceptional divisors being $E_{1}^{\prime \prime}, \ldots, E_{r}^{\prime \prime}$. Let $f^{\prime \prime}: \mathrm{Cl}\left(X^{\prime \prime}\right) \rightarrow \mathrm{Cl}(X)$ be the isometry defined by $f^{\prime \prime}(B)=B$, $f^{\prime \prime}(F)=F$ and $f^{\prime \prime}\left(E_{i}^{\prime \prime}\right)=E_{i}$ (where $B$ comes from the base curve and $F$ from a fiber of the ruling). Again we have $f^{\prime \prime}\left(M\left(X^{\prime \prime}\right)\right) \subset M(X)$.

Since $r=\rho(X)-2>n-2$, there is via elementary transformations a birational morphism $X^{\prime \prime} \rightarrow \mathbb{P}^{2}$. Hence $X^{\prime \prime}$ is a specialization of a blow-up $X^{\prime} \rightarrow \mathbb{P}^{2}$ at generic points. Let $f^{\prime}: \mathrm{Cl}\left(X^{\prime}\right) \rightarrow \mathrm{Cl}\left(X^{\prime \prime}\right)$ be the isometry given by the corresponding exceptional configurations in the usual way; thus $f^{\prime}\left(M\left(X^{\prime}\right)\right) \subset M\left(X^{\prime \prime}\right)$. We denote the composition $f^{\prime \prime} \circ f^{\prime}$ by $f: \mathrm{Cl}\left(X^{\prime}\right) \rightarrow \mathrm{Cl}(X)$. Using $f$, the argument now goes through as above.

We now can prove the main result of this section:

Corollary 4.2. Let $X$ be an anticanonical rational surface. Then $M(X)$ fails to be finitely generated if and only if either the set of-1-curves on $X$ or the set of -2-curves on $X$ is infinite.

Proof. The reverse implication is clear, since a prime divisor of negative selfintersection is not the sum of two nontrivial effective divisors (or see [Rosoff 1980, Fact, p. 426]). For the converse, apply Lemma 4.1 and Remark 2.4.

Here are some special cases of interest:

Proposition 4.3. Let $X$ be a rational surface.

(a) If $K_{X}^{2}>0$, then $M(X)$ is finitely generated.

(b) If $K_{X}^{2}=0$, then $M(X)$ is finitely generated if and only if $X$ has only finitely many-1-curves.

(c) If $K_{X}^{2}=0$ but $-K_{X}$ is not nef, $M(X)$ is finitely generated.

(d) If $K_{X}^{2}=0$ and $-K_{X}$ is nef, $M(X)$ is finitely generated if and only if the span of the -2-curves has rank $\rho(X)-1$ and $-K_{X}$ can be written as a positive rational linear combination of the -2-curves (all of them occurring).

(e) If $K_{X}^{2}<0$ and $X$ has a reduced irreducible anticanonical divisor, $M(X)$ is finitely generated if and only if $X$ has only finitely many -2-curves and the span of the -2-curves has rank $\rho(X)-1$. 
Proof. (a) This is well known, and follows from the purely intersection-formtheoretic fact that there are only finitely many -1 and -2 -classes. (Blow up points, if need be, so $K_{X}^{2}=1$. If there is a birational morphism $\pi: X \rightarrow \mathbb{P}^{2}$, see [Manin 1986]; if not, this applies anyway, using the isometry defined in Lemma 3.2.)

(b) One implication is clear. Conversely, it is enough to verify that there never can be infinitely many -2-curves. First suppose there is a birational morphism $X \rightarrow \mathbb{P}^{2}$; let $E_{0}, \ldots, E_{9}$ be the exceptional configuration. Consider a -2-class $D$. For some $r$, we have $\left(D+r K_{X}\right) \cdot E_{9}=0$, but it is easy to check that $D+r K_{X}$ is a -2-class, except now $D+r K_{X}$ is in the span of $E_{0}, \ldots, E_{8}$. By the proof of (a), there are only finitely many -2-classes in the span of $E_{0}, \ldots, E_{8}$. If there were infinitely many -2 -curves on $X$, two different ones, $A \neq B$, would have to be the same modulo $K_{X}$, and hence one, say $A$, would equal $B-m K_{X}$ for some $m>0$, which is impossible, since

$$
-2=A^{2}=A \cdot\left(B-m K_{X}\right)=A \cdot B>0 .
$$

By the usual isometry argument, even if there is no birational morphism to the plane, we would still have only finitely many -2 -classes modulo $K_{X}$, and the result again follows.

(c, d) These follow from [Lahyane 2001a; 2004], over $\mathbb{C}$, but the proofs work over any algebraically closed field.

(e) Note that there is a birational morphism $X \rightarrow \mathbb{P}^{2}$. (If not, $X$ is a blow-up of $\mathbb{F}_{n}$ for some $n>2$; hence $-K_{X} \cdot B<0$, so the proper transform $B^{\prime}$ of $B$ also meets $-K_{X}$ negatively. But $-K_{X}$ is irreducible, so $B^{\prime}$ must equal $-K_{X}$, which is not the case since $-K_{X}=2 B+(n+2) F-E_{1}-E_{2}-\cdots-E_{r}$ for some $r$, whereas $B^{\prime}$ is of the form $B$ minus some of the $E_{i}$ 's.) Moreover, any curve contracted by such a morphism must be a -1 or -2 -curve.

If $M(X)$ is finitely generated, there are only finitely many -1 and -2 -curves, hence only finitely many exceptional configurations. The span of the -2 -curves has rank $\rho(X)-1$, by [Harbourne 1985a, Theorem 3.1]. Conversely, if there are only finitely many -2 -curves and their span has rank $\rho(X)-1$, there are only finitely many exceptional configurations, also by [Harbourne 1985a, Theorem 3.1]. However, given a -1-curve $E$ on an anticanonical rational surface with a reduced irreducible anticanonical curve, the proof of [Harbourne 1985b, Theorem 2.1] gives a construction of an exceptional configuration $E_{0}, \ldots$, in which $E$ is $E_{i}$ for some $i>0$. There are only finitely many exceptional configurations, so there can be only finitely many -1 -curves $E$, and $M(X)$ is finitely generated by Corollary 4.2.

\section{Applications}

In this section, we give examples of rational surfaces $X$ with an infinite number of -1-curves. By [Nagata 1960a, Theorem 5], there is a birational morphism $X \rightarrow \mathbb{P}^{2}$ 
for any such $X$; hence each of our examples $X$ has an exceptional configuration, $E_{0}, \ldots, E_{n}$. In all but Example 5.5, we will have $n=9$. We remind the reader of the notation $s_{i}$ from Remark 2.8 for certain particular -2-classes.

Example 5.1. This example provides a configuration of -2-curves whose intersection graph is of type $E_{8}$. Let $p$ be a flex of a smooth plane cubic curve $C$. Blow up eight times, starting at $p$ and continuing with the seven successive points of $C$ infinitely near $p$; hence $s_{0}, \ldots, s_{7}$ are the classes of -2 -curves. Then blow up a ninth point $q \in C$ such that $p-q$ does not have finite order in the divisor class group of $C$. Then $s_{0}, \ldots, s_{7}$ give the only -2 -curves on the resulting surface $X$. (This is because, by [Harbourne 1985a, Lemma 1.3 and p. 136] or [Looijenga 1981], any -2-curve $N$ is a nonnegative integer linear combination of $s_{0}, \ldots, s_{8}$ which restricts trivially to the proper transform $C^{\prime}$ of $C$. Since $\mathbb{O}_{C^{\prime}}\left(s_{8}\right)=\mathscr{O}_{C^{\prime}}(p-q)$, this combination cannot involve $s_{8}$, so now we see that the combination must be one of $s_{0}, \ldots, s_{7}$, since $N$ is irreducible.) Hence $M(X)$ is not finitely generated by Proposition 4.3(d), and, by Proposition 3.3, $E_{9}+d\left(s_{8}+K_{X}\right)-d^{2} K_{X}$ is the class of a -1-curve on $X$ for each positive integer $d$.

Example 5.2. This example provides a configuration of -2-curves of type $A_{1} E_{7}$. Let $p$ and $q$ be as in the previous example, but blow up only 7 times at $p$ and twice at $q$. Arguing as in the previous example, we see that the only-2-curves on $X$ are $s_{0}, \ldots, s_{6}$ and $s_{8}$. As before, $M(X)$ is not finitely generated, and this time, for each positive integer $d, E_{9}+d s_{7}-d^{2} K_{X}$ is the class of a-1-curve.

Example 5.3. This example provides a configuration of -2-curves of type $A_{1} A_{7}$. This time choose $p$ and $q$ on a smooth elliptic curve $C$ such that $p-q$ has infinite order, and embed $C$ in the plane using $|2 p+q|$. Neither $p$ nor $q$ is a flex and the line tangent to $C$ at $p$ goes through $q$. Blow up twice at $p$, and then seven times at $q$. Arguing as before, the -2 -curves are $s_{0}, s_{1}$ and $s_{3}, \ldots, s_{8}$, again $M(X)$ is not finitely generated, and for each positive integer $d, E_{9}+d s_{2}-d^{2} K_{X}$ is a -1-curve.

Example 5.4. This example provides a configuration of -2-curves of type $2 A_{1} D_{6}$. This time, start with an abstract elliptic curve $C$ such that $\operatorname{Pic}^{0}(C)$ contains a subgroup isomorphic to $\mathbb{Z} / 2 \mathbb{Z} \times \mathbb{Z}$. Let $p_{1}$ be any point of $C$, but pick $p_{2}$ and $q$ such that $p_{1}-p_{2}$ is a generator for $\mathbb{Z} / 2 \mathbb{Z}$ and $p_{1}-q$ is a generator for $\mathbb{Z}$. Embed $C$ in the plane via $\left|2 p_{1}+q\right|$. By construction, the line tangent to $C$ at either $p_{1}$ or $p_{2}$ passes through $q$. Now blow up 6 times on $C$ at $p_{1}$, then twice at $p_{2}$ and once at $q$. The resulting -2 -curves are $s_{1}, \ldots, s_{5}, s_{7}, E_{0}-E_{1}-E_{2}-E_{9}$, and $E_{0}-E_{7}-E_{8}-E_{9}$. Then $M(X)$ is not finitely generated, and for each positive integer $d, E_{9}+d\left(E_{9}-K_{X}-E_{1}\right)-d^{2} K_{X}$ is the class of a -1-curve on $X$.

Example 5.5. The set of -2-curves can be infinite, and their span can even have rank $\rho(X)-1$; see [Harbourne 1985a, Note 3.3] for an example with $\rho(X)=11$. In this example, it turns out that there are also infinitely many -1-curves. This raises several questions. Can it ever happen that there are infinitely many -2-curves but 
only finitely many -1 -curves, or is $M(X)$ not finitely generated if and only if there are infinitely many -1 -curves? Does $M(X)$ failing to be finitely generated always imply the existence of a birational morphism $X \rightarrow \mathbb{P}^{2}$ ? What can be said about these questions if $X$ is rational but not necessarily anticanonical?

Example 5.6. We show in case $K_{X}^{2}=0$ that $M(X)$ can fail to be finitely generated although the span of the -2 -curves has rank 9 . For specificity and simplicity, we work over the complex numbers, but with suitable changes, similar examples can be obtained over any algebraically closed field. Pick three distinct points $p, q, r$ on a line $L$ in the plane. Choose an affine coordinate $t$ on $L$ such that $t=0$ is $p$, $t=-1$ is $q$ and $t=1$ is $r$. Choose a fourth point $s$ whose value of $t$ is such that

$$
\left(\frac{t}{t+1}\right)^{m} \neq\left(\frac{1}{2}\right)^{m}
$$

for all positive integers $m$. Thus $t /(t+1)$ has a zero at $p$ and a pole at $q$, but no power of $t /(t+1)$ ever takes on the same value at $r$ and $s$. Now pick a smooth conic $C$ that meets $L$ at $r$ and $s$, and choose a point $c$ on $C$ such that the line through $c$ and $q$ is tangent to $C$ at $c$. Blow up the plane at the point $c$, and then again at the point of the proper transform of $C$ infinitely near to $c$, and continue in this way until you have blown up six times. Thus the proper transform $C^{\prime}$ of $C$ is a -2curve. Similarly, blow up three times on $L$ at $p$, so the proper transform $L^{\prime}$ of $L$ is a -2 -curve. Let $X$ denote the plane blown up at the nine given points. In terms of the sequence of blow-ups just specified, we have an exceptional configuration $E_{0}$, $\ldots, E_{9}$ with respect to which $C^{\prime}=2 E_{0}-E_{1}-\cdots-E_{6}, L^{\prime}=E_{0}-E_{7}-E_{8}-E_{9}$, and $s_{1}, \ldots, s_{5}, s_{7}$ and $s_{8}$ are -2 -curves.

Suppose $N$ is a-2-curve other than $L^{\prime}$ or $C^{\prime}$. Then $N$, as before, is a nonnegative integer linear combination of $s_{0}, \ldots, s_{8}$ whose restriction to $L^{\prime}+C^{\prime}=-K_{X}$ is trivial. For the restriction to be trivial, we must of course have $N \cdot L^{\prime}=0$ and $N \cdot C^{\prime}=0$, and in addition we need $0_{L^{\prime}+C^{\prime}}(N)=0$. But $s_{0}, \ldots, s_{8}$ form a basis of $K_{X}^{\perp}$, and each restricts trivially to $L^{\prime}+C^{\prime}$ except that

$$
\mathcal{O}_{L^{\prime}+C^{\prime}}\left(s_{0}\right)=\mathcal{O}_{L^{\prime}+C^{\prime}}(q-c) \text { and } \mathcal{O}_{L^{\prime}+C^{\prime}}\left(s_{6}\right)=\mathcal{O}_{L^{\prime}+C^{\prime}}(c-p) .
$$

Thus for any $N$ with $N \cdot L^{\prime}=0$ and $N \cdot C^{\prime}=0$ we have

$$
\mathcal{O}_{L^{\prime}+C^{\prime}}(N)=\mathfrak{O}_{L^{\prime}+C^{\prime}}(m(p-q)) .
$$

Suppose this is trivial for some $m$. If $m=0$, then $N$ is a combination of only $s_{1}, \ldots, s_{5}, s_{7}$ and $s_{8}$, and being irreducible $N$ must be one of $s_{1}, \ldots, s_{5}, s_{7}$ and $s_{8}$. If $m \neq 0$, we may assume $m>0$, in which case there must be a rational function on $L$ with a zero of order $m$ at $p$ and a pole of order $m$ at $q$, taking on the same values at $r$ and $s$ (and so extending to $L+C$ ). By construction, there is no such rational function on $L$. Thus there are no -2-curves beyond the nine already noted, whose span has rank 9 . On the other hand, $E_{7}-E_{9}$ is in $K_{X}^{\perp}$, meets every -2-curve nonpositively, and meets $s_{7}$ (and $s_{8}$ for that matter) negatively. Thus 
$-K_{X}$ cannot be a rational linear combination of the -2 -curves with all positive coefficients. Thus Proposition 4.3(d) implies $M(X)$ is not finitely generated. Here $E_{9}+d\left(E_{9}-E_{7}-K_{X}\right)-d^{2} K_{X}$ is the class of a -1 -curve on $X$, for each positive integer $d$.

\section{References}

[Alberich-Carramiñana] M. Alberich-Carramiñana, "Plane Cremona maps, exceptional curves and roots", Trans. Amer. Math. Soc. To appear.

[Harbourne 1985a] B. Harbourne, "Blowings-up of $\mathbf{P}^{2}$ and their blowings-down", Duke Math. J. 52:1 (1985), 129-148. MR 86m:14026 Zbl 0577.14025

[Harbourne 1985b] B. Harbourne, "Complete linear systems on rational surfaces", Trans. Amer. Math. Soc. 289:1 (1985), 213-226. MR 86h:14030 Zbl 0609.14004

[Harbourne 1997] B. Harbourne, "Anticanonical rational surfaces", Trans. Amer. Math. Soc. 349:3 (1997), 1191-1208. MR 97f:14007 Zbl 0860.14006

[Hartshorne 1977] R. Hartshorne, Algebraic geometry, Graduate Texts in Mathematics 52, Springer, New York, 1977. MR 57 \#3116

[Kac 1985] V. G. Kac, Infinite-dimensional Lie algebras, 2nd ed., Cambridge University Press, Cambridge, 1985. MR 87c:17023

[Lahyane 2001a] M. Lahyane, "Exceptional curves on smooth rational surfaces with $-K$ not nef and of self-intersection zero", preprint, ICTP (Trieste), 2001, Available at http://www.ictp.trieste.it/ $\sim$ pub_off/preprints-sources/2001/IC2001105P.tex.

[Lahyane 2001b] M. Lahyane, "Rational surfaces having only a finite number of exceptional curves", preprint IC2001141, ICTP (Trieste), 2001, Available at http://www.ictp.trieste.it/ pub_off.

[Lahyane 2004] M. Lahyane, "Rational surfaces having only a finite number of exceptional curves", Math. Z. 247:1 (2004), 213-221. MR 2054527

[Looijenga 1981] E. Looijenga, "Rational surfaces with an anticanonical cycle", Ann. of Math. (2) 114:2 (1981), 267-322. MR 83j:14030 Zbl 0509.14035

[Manin 1986] Y. I. Manin, Cubic forms: Algebra, geometry, arithmetic, 2nd ed., North-Holland Mathematical Library 4, North-Holland, Amsterdam, 1986. MR 87d:11037 Zbl 0582.14010

[Nagata 1960a] M. Nagata, "On rational surfaces, I: Irreducible curves of arithmetic genus 0 or 1", Mem. Coll. Sci. Univ. Kyoto Ser. A Math. 32 (1960), 351-370. MR 23 \#A3739 Zbl 0100.16703

[Nagata 1960b] M. Nagata, "On rational surfaces, II", Mem. Coll. Sci. Univ. Kyoto Ser. A Math. 33 (1960), 271-293. MR 23 \#A3740 Zbl 0100.16801

[Rosoff 1980] J. A. Rosoff, "Effective divisor classes and blowings-up of $\mathbf{P}$ ", Pacific J. Math. 89:2 (1980), 419-429. MR 82e:14042

[Rosoff 1981] J. A. Rosoff, "The monoid of effective divisor classes of a complex torus", pp. 208231 in Algebraic geometry (Chicago, 1980), edited by A. Libgrober and P. Wagreich, Lecture Notes in Math. 862, Springer, Berlin, 1981. MR 83m:14032 Zbl 0488.14002

[Rosoff 2002] J. Rosoff, "Effective divisor classes on a ruled surface", Pacific J. Math. 202:1 (2002), 119-124. MR 2003a:14055 Zbl 01818940

Received August 27, 2001. Revised June 1, 2004. 


\author{
Mustapha Lahyane \\ Departamento Álgebra, Geometría y Topología \\ FACULTAD DE CIENCIAS \\ UNIVERSIDAD DE VALLADOLID \\ C/ PRADo DE LA MAGDalena S/N \\ E-47005 VALLADOLID \\ SPAIN \\ lahyane@agt.uva.es \\ BRIAN HARBOURNE \\ DEPARTMENT OF MATHEMATICS \\ UNIVERSITY OF NEBRASKA \\ LINCOLN, NE 68588-0323 \\ UNITED STATES \\ bharbour@math.unl.edu \\ http://www.math.unl.edu/ bharbour
}

